\title{
Editorial for Special Issue on ICONIP 2014
}

\author{
John Sum ${ }^{1}$ • Andrew C. S. Leung ${ }^{2}$
}

Published online: 22 April 2016

C) Springer Science+Business Media New York 2016

It has been 21 years since the first International Conference on Neural Information Processing (ICONIP) held in Seoul, Korea, and now is the annual conference of the Asia Pacific Neural Network Assembly (APNNA) aiming at providing a platform for the neural processing community to present their researches and to exchange the research ideas. In 2014, ICONIP was held in Sarawak, Malaysia, during November 3-6, 2014. Around 370 submissions, from 30 countries and regions across six continents, were received and each article was evaluated by at least two qualified reviewers. Finally, around 230 high-quality papers were accepted for oral presentations.

This special issue aims at disseminating the latest development of neural information processing and machine learning. Six extended papers from ICONIP 2014 are included in this issue and each of them has gone through an additional peer review process. They were evaluated by at least two additional qualified reviewers. We include the papers with the best representation and achievement in their respective fields:

(1) "Sparse and Truncated Nuclear Norm based Tensor Completion" proposes a norm for tensor data. Furthermore a tensor completion algorithm is developed based on the concept of the alternating direction method of multipliers (ADMM). To maintain the structural information, a sparse regularization term, defined in the transform domain, is added into the objective function.

(2) "Online Depth Image-based Object Tracking with Sparse Representation and Object Detection" addresses the object tracking problem in the presence of occlusion and illumination change. With sparse coding representation for input images, the proposed method is able to keep tracking the target object with high occlusion area.

(3) "Visual Salience Using Binary Spectrum of Walsh-Hadar transform (WHT) and Its Applications to Ship Detection in Multicultural Imagery" addresses the visual saliency detection problem. Based on the WHT, the proposed method projects the input image

\section{John Sum}

pfsum@yahoo.com.hk

1 National Chung Hsing University, Taichung, Taiwan

2 City University of Hong Kong, Kowloon Tong, Hong Kong 
into the WHT space and utilizes the sign information of the WHT coefficients to estimate the saliency information. Compared to the conventional methods, the proposed method can significantly reduce the computational loading.

(4) "Cross-modal Salience Correlation for Image Annotation" proposed a Textual-Visual Salience based Annotation (TVSA) method for image annotation. First, both textual saliency map (a graph) and community visual saliency maps (multiple graphs each corresponds to one community) are generated. Then, the maps are used for training a multiple-kernel SVM classifier. The classifier could thus be applied to generate annotations to an image.

(5) "Joint Learning of Unsupervised Dimensionality Reduction and Gaussian Mixture Model", considers a joint optimization procedure for reducing data dimension and estimating the parameters of Gaussian mixture model. By modeling the data distribution as a special Gaussian mixture model, the mixture of factor analyzer with common factor loading (MCFA), the authors present an expectation-maximization (EM) algorithm to estimate the model parameters with a fix number of latent variables. Applying the algorithm to different number of latent variables, the best model with reduced dimensionality could be obtained.

(6) "Directed Connectivity Analysis of Functional Brain Networks during Cognitive Activity using Transfer Entropy" studies the information flow in functional brain networks during cognitive activity. The paper utilizes the concept of normalized transfer entropy to construct a directed functional brain network. Furthermore, it explores graph theoretic and statistical analysis to characterize the constructed directed FBN and the information flow pattern of the constructed directed FBN during cognitive tasks.

We would like to thank all the authors and reviewers who contributed their time and effort for this special issue. Besides, we are grateful to all those people behind this endeavor, in particular the Springer JET assistant Nagasakthi Murmansk who assisted us in various aspects. 